\title{
Inhaled and intravenous application of a stimulator of the soluble guanylate cyclase (BAY 41-8543) reduces pulmonary vascular resistance in a model of septic shock
}

Nils Kronas, Birte Peters, Alwin E Goetz, Jens C Kubitz

From 5th International Conference on CGMP: Generators, Effectors and Therapeutic Implications

Halle, Germany. 24-26 June 2011

\section{Background}

Cardiac failure in septic shock usually originates from hypovolaemia, impaired global contractility or right ventricular failure (RVF). Acute RVF due to endotoxinmediated pulmonary hypertension results in global hypoperfusion. However, the treatment of pulmonary vascular resistance (PVR) in septic patients remains a black box.

\section{Methods}

After ethical approval, septic shock was induced in 32 pigs ( $25 \pm 3 \mathrm{~kg}$ ) by continuous infusion of endotoxin (Escherichia coli serotype 0111:B4). The animals received a protocol-based treatment with fluids and vasopressors according to the surviving sepsis campaign guidelines. Then, they were randomized to either inhaled (i.h.) $\left(240 \mu \mathrm{g} \mathrm{kg}^{-1}\right)$ or intravenous (i.v.) $(24 \mu \mathrm{g} \mathrm{kg}$ $\left.{ }^{1}\right)$ treatment with BAY 41-8543 or controls. Heart rate (HR (bpm)), mean arterial pressure (MAP (mmHg)), mean pulmonary artery pressure (MPAP $(\mathrm{mmHg}))$, cardiac output $\left(\mathrm{CO}\left(\mathrm{l} \mathrm{min}^{-1}\right)\right)$ and PVR (dyn sec $\left.\mathrm{cm}^{-5}\right)$ were assessed every 15 minutes for $1 \mathrm{~h}$ after starting the treatment. Following a wash out period of $1 \mathrm{~h}$, the animals were once more randomized to double dose (D2) i. h. or i.v. respectively or to simultaneous administration of Bay 41-8543 at a single dose together with NO (i.h.) at $20 \mathrm{ppm}$. Hemodynamic measurements were taken for another hour. Data are expressed as mean \pm SD at

Table 1

\begin{tabular}{|c|c|c|c|c|c|c|c|c|c|}
\hline & $\begin{array}{l}\text { shock } \\
(n=32)\end{array}$ & $\begin{array}{c}\text { i.h. } \\
(n=12)\end{array}$ & $\begin{array}{c}\text { i.v. } \\
(n=14)\end{array}$ & $\begin{array}{c}\text { con } \\
(n=6)\end{array}$ & $\begin{array}{c}\text { i.h. D2 } \\
(n=6)\end{array}$ & $\begin{array}{c}\text { i.h. no } \\
(n=5)\end{array}$ & $\begin{array}{c}\text { i.v. D2 } \\
(n=5)\end{array}$ & $\begin{array}{c}\text { i.v. no } \\
(n=5)\end{array}$ & $\begin{array}{c}\text { con no } \\
(n=5)\end{array}$ \\
\hline HR & $\begin{array}{l}127 \\
(34)\end{array}$ & $\begin{array}{l}150 \\
(21)\end{array}$ & $\begin{array}{c}163^{* * *} \\
(28)\end{array}$ & $\begin{array}{l}123 \\
(34)\end{array}$ & $\begin{array}{l}140 \\
(35)\end{array}$ & $\begin{array}{l}146 \\
(19)\end{array}$ & $\begin{array}{l}171 \\
(29)\end{array}$ & $\begin{array}{l}148 \\
(29)\end{array}$ & $\begin{array}{l}113 \\
(34)\end{array}$ \\
\hline MAP & $\begin{array}{l}58 \\
(7)\end{array}$ & $\begin{array}{l}71 \\
(7)\end{array}$ & $\begin{array}{l}68 \\
(6)\end{array}$ & $\begin{array}{l}73 \\
(8)\end{array}$ & $\begin{array}{l}75 \\
(6)\end{array}$ & $\begin{array}{l}69 \\
(9)\end{array}$ & $\begin{array}{l}68 \\
(14)\end{array}$ & $\begin{array}{c}73 \\
(10)\end{array}$ & $\begin{array}{l}73 \\
(8)\end{array}$ \\
\hline MPAP & $\begin{array}{l}38 \\
(7)\end{array}$ & $\begin{array}{l}40 \\
(7)\end{array}$ & $\begin{array}{l}37 \\
(8)\end{array}$ & $\begin{array}{l}44 \\
(6)\end{array}$ & $\begin{array}{c}42 \\
(10)\end{array}$ & $\begin{array}{l}42 \\
\text { (9) }\end{array}$ & $\begin{array}{c}40 \\
(16)\end{array}$ & $\begin{array}{c}37^{* *} \\
(9)\end{array}$ & $\begin{array}{c}39^{* *} \\
(9)\end{array}$ \\
\hline PVR & $\begin{array}{l}1246 \\
(401)\end{array}$ & $\begin{array}{c}491^{* *} \\
(106)\end{array}$ & $\begin{array}{c}469^{* *} \\
(198)\end{array}$ & $\begin{array}{c}972 \\
(325)\end{array}$ & $\begin{array}{l}691^{* *} \\
(322)\end{array}$ & $\begin{array}{l}573^{* *} \\
(245)\end{array}$ & $\begin{array}{l}849^{*} \\
(815)\end{array}$ & $\begin{array}{l}460^{* *} \\
(103)\end{array}$ & $\begin{array}{l}887^{* *} \\
(296)\end{array}$ \\
\hline $\mathrm{CO}$ & $\begin{array}{c}2.02 \\
(0.51)\end{array}$ & $\begin{array}{c}4.65^{* *} \\
(0.76)\end{array}$ & $\begin{array}{l}5.04^{* *} \\
(1.43)\end{array}$ & $\begin{array}{c}2.93 \\
(0.89)\end{array}$ & $\begin{array}{c}3.73 \\
(1.71)\end{array}$ & $\begin{array}{l}4.16^{*} \\
(1.44)\end{array}$ & $\begin{array}{l}4.3^{* *} \\
(2.85)\end{array}$ & $\begin{array}{c}4.06^{* *} \\
(0.78)\end{array}$ & $\begin{array}{c}2.46 \\
(0.67)\end{array}$ \\
\hline
\end{tabular}

$* p<0.05$

${ }^{* *} p<0.01$

vs. control group (con)

*Correspondence: jkubitz@uke.de

Department of Anesthesiology, University of Hamburg, Hamburg, Germany

(C) 2011 Kronas et al; licensee BioMed Central Ltd. This is an open access article distributed under the terms of the Creative Commons 
shock and 30 minutes after treatment. Differences between groups were analyzed using a linear mixedeffects model.

\section{Results}

Both during i.h. and i.v. application of BAY 41-8543, a significant decrease in PVR and an increase in CO was observed. Additive inhaled NO decreased PVR more than doubling the dose of BAY 41-8543 (Table 1).

\section{Conclusion}

The stimulator of the soluble guanylate cyclase BAY 418543 offers a treatment option for pulmonary hypertension in septic shock. Both inhalative and intravenous administration of BAY 41-8543 reduces PVR and increase $\mathrm{CO}$. Further, there seems to be an additive effect of inhaled NO.

Published: 1 August 2011

doi:10.1186/1471-2210-11-S1-P41

Cite this article as: Kronas et al:: Inhaled and intravenous application of a stimulator of the soluble guanylate cyclase (BAY 41-8543) reduces

pulmonary vascular resistance in a model of septic shock. BMC

Pharmacology 2011 11(Suppl 1):P41.
Submit your next manuscript to BioMed Central and take full advantage of:

- Convenient online submission

- Thorough peer review

- No space constraints or color figure charges

- Immediate publication on acceptance

- Inclusion in PubMed, CAS, Scopus and Google Scholar

- Research which is freely available for redistribution

Submit your manuscript at www.biomedcentral.com/submit 